\title{
Ring fiber with flat-top profile for extremely large mode area single mode operation
}

\author{
Deepak Jain* and Jayanta K. Sahu \\ Optoelectronics Research Center, University of Southampton, UK \\ e-mail address: dj3g11@orc.soton.ac.uk
}

\begin{abstract}
We propose novel optical fiber for extremely large mode area with flat-top output beam. Numerical simulations show a record effective-area larger than $11,000 \mu \mathrm{m}^{2}$ from just a $58 \mu \mathrm{m}$ diameter core with flat-top output in rod-type configuration.

OCIS codes: (060.2280) Fiber design and manufacturing; (140.3510) Fiber lasers.
\end{abstract}

\section{Introduction}

High power fiber laser have seen dramatic progress in the last decade, thanks to their outstanding features like good beam quality, better thermal management, flexibility, high wall-plug efficiency, and low foot print etc. Fiber lasers have already crossed the landmark of $10 \mathrm{~kW}$ output power in continuous wave regime and $3.8 \mathrm{GW}$ peak power in pulse regime with Gaussian output, utilizing the single mode operation of large mode area fiber designs [1]. However, bend induced detrimental mode area reduction can dramatically influence the advantages achieved from large mode area. In order to avoid bend induced effects, a rod-type fiber configuration can be used, which typically has few mm thicknesses and cannot be bent [2]. Rod-type fiber design based on higher-order-mode (HOM) suppression have been proposed, such as large pitch fiber (LPF) [2], distributed modal filtering fiber (DMF) [3], and multi trench fiber (MTF) [4]. These fiber designs can achieve a Gaussian output profile with effective area $10,000 \mu \mathrm{m}^{2}$ to $12,000 \mu \mathrm{m}^{2}$ from a $\sim 130-140 \mu \mathrm{m}$ diameter core fiber. In this paper, we propose a novel fiber design, which can achieve extremely large mode area single mode operation with flat top output beam. Here we report, a record effective area $\left(>11,000 \mu \mathrm{m}^{2}\right)$ for a flat-top output profile from a small core diameter $(\sim 58 \mu \mathrm{m})$. The proposed fiber design is an all-solid structure and is simple to realize.

\section{Fiber design and working principle}

Figure 1(a) shows the schematic of refractive index profile (RIP) of the proposed ring fiber and 1(b) shows the 2-D cross-section of fiber end. Fiber has only one high index ring and the thickness of ring is $t$ and refractive index of ring w.r.t surrounding is $\Delta \mathrm{n}$. The region covered by high index ring is core and have a radius rc. In this design, the ring is the only high refractive index element. Hence, it is ring that guides the light.

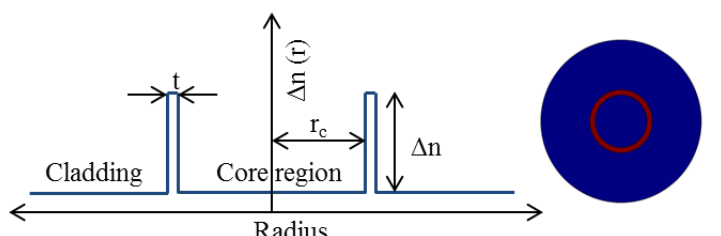

(a)

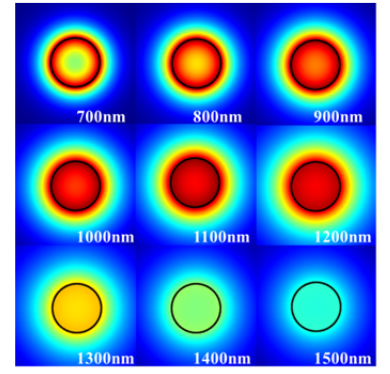

(d)

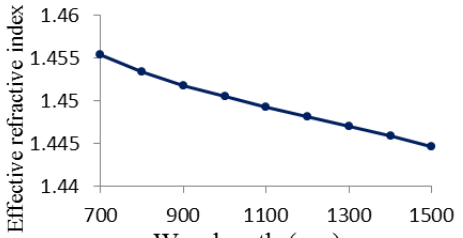

(c) Wavelength $(\mu \mathrm{m})$

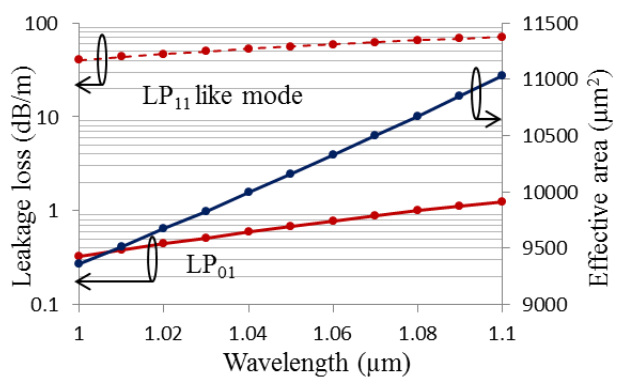

(e)

Fig. 1(a) Schematic of RIP of proposed ring fiber (b) 2-D cross-section of fiber (c) effective refractive index of FM w.r.t. wavelength (d) normalized electric field pattern for FM w.r.t different wavelength (e) leakage loss for FM and LP11 like mode and effective area of FM for $\mathrm{rc}=29 \mu \mathrm{m}, \mathrm{t}=1 \mu \mathrm{m}$, and $\Delta \mathrm{n}=0.0005$. 
We performed a finite element method based numerical analysis of the proposed optical fiber using the commercially available COMSOL software. Fig. 1(c) shows the computed effective refractive index of the fundamental mode (FM) with respect to wavelength for $\mathrm{rc}=29 \mu \mathrm{m}, \mathrm{t}=1 \mu \mathrm{m}$, and $\Delta \mathrm{n}=0.0005$. Fig. 1 (d) shows the normalized electric field of FM at different wavelengths. It is interesting to note that at shorter wavelength, FM looks like a ring mode and with increasing wavelength the effective refractive index of mode decreases and it appears like a flat-top mode, thanks to the spreading electric field in the core region with increasing wavelength [5]. This flat-field leads to a very large effective area for the FM. It is worth citing here that an effective area larger than $11,000 \mu \mathrm{m}^{2}$ can be achieved from a $58 \mu \mathrm{m}$ effective core size, as shown in Fig. 1(e), which is dramatic progress compared to Gaussian field profile from a similar core size. In order to achieve a $11,000 \mu \mathrm{m}^{2}$ effective area, a core diameter larger than $100 \mu \mathrm{m}$ in rod-type configuration is needed, where a Gaussian field profile is employed [2, 4].

\section{Single mode behaviour and delocalization of higher order modes}

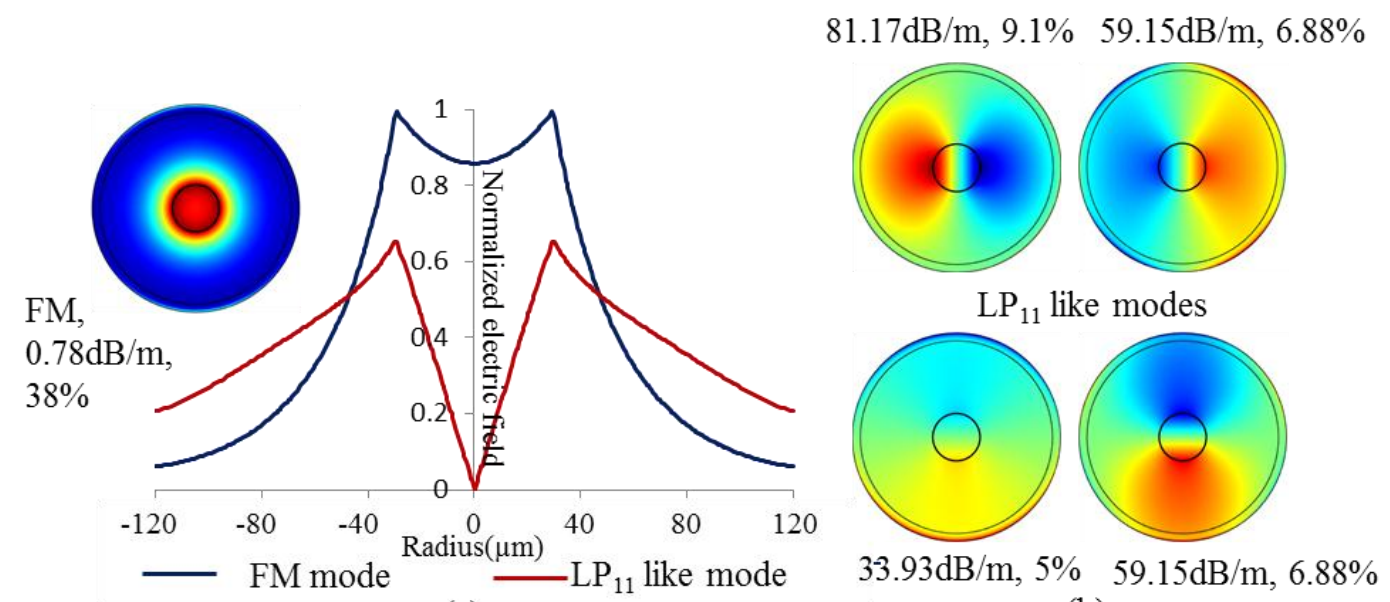

(a)

(b)

Fig. 2(a) normalized electric field for FM and $\mathrm{LP}_{11}$ like mode. Inset also shows the 2-d normalized electric field pattern of FM. (b) transverse electric field of $\mathrm{LP}_{11}$ like modes.

Figure 2 shows the normalized electric field of the $\mathrm{FM}$ and $\mathrm{LP}_{11}$ like modes for $\mathrm{rc}=29 \mu \mathrm{m}, \mathrm{t}=1 \mu \mathrm{m}$, and $\Delta \mathrm{n}=0.0005$. The $\mathrm{LP}_{11}$ like modes are extremely leaky and have very high loss $(>30 \mathrm{~dB} / \mathrm{m})$ due to their very low effective refractive index, while FM mode loss is reasonably low $(<1 \mathrm{~dB} / \mathrm{m})$. We did not observe any HOM other than $\mathrm{LP}_{11}$. The effective area of the FM is approximately $11,120 \mu \mathrm{m}^{2}$. However, in a double clad configuration, which is suitable for fiber laser, effective leakage loss of HOMs is not the sole criterion for single mode operation. Therefore, a discrimination based on overlap with the doped core region between FM and HOM is another necessary condition to ensure a single-mode operation. In this case, we see a 38\% overlap of FM power with core doped region, on other hand it is lower than $10 \%$ for other higher order modes. For the same suppression of the HOMs (by leakage loss and power discrimination), the effective area of the proposed design is much larger than that obtained from competitive designs such as LPF, MTF, and DMF, thanks to the flat-field distribution.

Figure 3(a) shows the effective area and leakage loss of the FM for different core size diameter such as $38 \mu \mathrm{m}$, $48 \mu \mathrm{m}, 58 \mu \mathrm{m}$, and $68 \mu \mathrm{m}$. An effective area as large as $12,332 \mu \mathrm{m}^{2}$ can be achieved from $68 \mu \mathrm{m}$ core diameter while the leakage loss of the FM remains lower than $1 \mathrm{~dB} / \mathrm{m}$. On the other hand, Fig. 3(b) shows the power fraction of the FM and other HOMs. We classify the HOMs in two categories here; (1) HOM having lowest loss and (2) HOM having highest power fraction in core. The loss and power fraction for both of these categories have been shown in Fig. 3(b). Fiber maintains a good discrimination between FM and HOM for all the cases. However, this discrimination either in term of loss or power fraction decreases with increasing core size. In case of $38 \mu \mathrm{m}$, we did not observe any HOM. Hence, the loss and power fraction is not being reported here. For $48 \mu \mathrm{m}$ core diameter, HOM loss is higher than $41 \mathrm{~dB} / \mathrm{m}$ and power fraction is lower than $6.2 \%$, while the effective area of the FM is $\sim 7381 \mu \mathrm{m}^{2}$. In case of $58 \mu \mathrm{m}$ core, $\mathrm{HOM}$ loss is higher than $33 \mathrm{~dB} / \mathrm{m}$ and power fraction is lower than $9.2 \%$, while the effective area of the FM is $\sim 11,120 \mu \mathrm{m}^{2}$. On other hand, in case of $68 \mu \mathrm{m}$ core diameter fiber, HOM loss is higher than $13 \mathrm{~dB} / \mathrm{m}$ and power fraction is lower than $15.5 \%$, while the effective area of the FM is $\sim 12,332 \mu \mathrm{m}^{2}$. It is 
important to note that the HOM having $13 \mathrm{~dB} / \mathrm{m}$ loss have only $10.83 \%$ power fraction in core, while the HOM having $15.44 \%$ power fraction in core have more than $55 \mathrm{~dB} / \mathrm{m}$ loss.

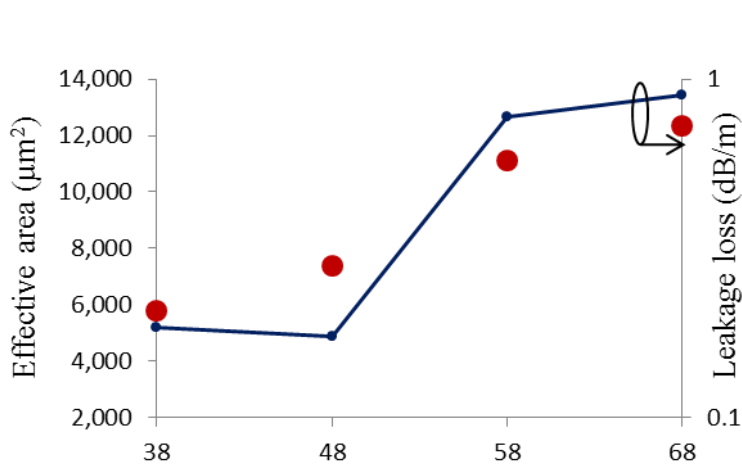

(a) Core diameter $(\mu \mathrm{m})$

- Effective area Leakage loss

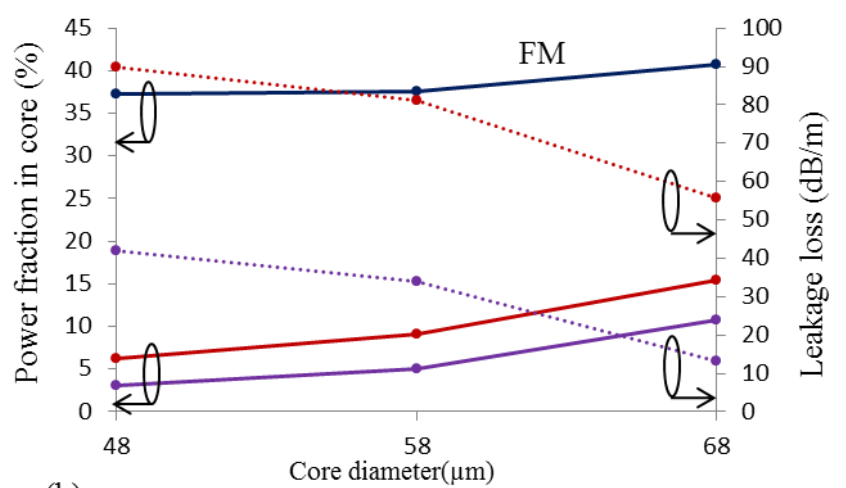

(b) Power fraction of HOM having lowest loss

Power fraction of HOM having highest power fraction in core Loss of HOM having lowest loss Loss of HOM having highest power fraction in core

Fig. 3 (a) Effective area and leakage loss of the FM for different core size diameter (b) Power fraction and leakage for the FM, HOM having lowest loss, and HOM having highest power fraction for different core size diameter. Here $\mathrm{t}=1 \mu \mathrm{m}, \Delta \mathrm{n}=0.0006$ for $38 \mu \mathrm{m}$ and $48 \mu \mathrm{m}$ core diameter and $\Delta \mathrm{n}=0.0005$ for $58 \mu \mathrm{m}$ and $68 \mu \mathrm{m}$ core diameter.

Our proposed fiber design has the advantage of cylindrical symmetry, thus it is relatively simple to fabricate. It can be fabricated using conventional chemical vapour deposition (CVD) process such as MCVD, PECVD, and OVD etc. However, challenges of fabricating index-compensated rare-earth doped core still remains there. It is interesting to see that due to the flat-top profile, it can also be used for several material processing applications such as welding, drilling, cutting, marking etc, where Gaussian beam profile is not good enough. PCF based designs with a flat-top output beam profile have been reported previously [5-6]. However, these designs are difficult to fabricate and reported area is lower than $2,000 \mu \mathrm{m}^{2}$. To the best of our knowledge, this is the record effective area with flat-top profile from a small core diameter. It is important to understand that flat-top profile is difficult to achieve in a bent fiber as the effective refractive index profile is really different in bent case compared to unbent case. Moreover, in bent case the effective area in flat-top design suffers more reduction than a normal step-index fiber. In order to exploit the flat-top profile to avoid non-linear effect by increasing the effective area, it is best to use them in rodtype configuration.

\section{Conclusion}

We have proposed a novel fiber design, which provides a flat-top output beam and thus leads to extremely large mode area. Numerical simulation shows the possibility of achieving effective area as large as $12,000 \mu \mathrm{m}^{2}$ with very high suppression of higher order modes, from a core diameter of just $58 \mu \mathrm{m}$. Fiber design offers certain advantages as of all-solid structure and cylindrical symmetrical design. To the best of our knowledge, this is the simplest design ever reported for extremely large mode area and record effective area for flat-top profile with numerous applications.

\section{References}

[1] C. Jauregui et. al., "High-power fiber lasers," Review, Nature Photonics, vol. 7, 861-867, 2013.

[2] J. Limpert et. al., "Yb-doped large-pitch fibers: effective single-mode operation based on higher-order mode delocalization", Light: Sci. \& App., 1, 2012.

[3] M. Laurila et. al., "Distributed mode filtering rod fibre amplifier delivering 292W with improved mode stability," Opt. Express 20, 57425753,2012 .

[4] D. Jain, et. al., "Mode area scaling with Multi-trench rod-type fibers," Opt. Exp. 21, 1448-1455, 2013.

[5] C. Valetin et. al., "Top-hat beam output output of a single-mode microstructured optical fiber: Impact of core index depression,” Opt. Exp. 21, 23250-23260, 2013.

[6] F. Kong et. al., "Flat-top mode from a 50 m-core Yb-doped leakage channel fiber," Opt. Exp. 21, 32371-32376, 2013. 